\section{Auditory signal detection: Effects of long-term practice and time on task}

\author{
GERARD A. KERKHOF, \\ TJERK W. van der SCHAAF, and H. JAN KORVING \\ Department of Physiology and Department of Psychology \\ State University of Leiden, Leiden, The Netherlands
}

Within the context of the theory of signal detectability (TSD; Green \& Swets, 1966), only a few studies report on the effects of practice and time on task.

Green and Swets (1966, p. 399) conclude, on the basis of the studies by Gundy (1961), Lukaszewski and Elliot (1962), Swets and Sewall (1963), and Zwislocki, Maire, Feldman, and Rubin (1958), that as far as such simple signals as pure tones are concerned, any practice effect is limited to the first experimental session and consequently is not a serious concern. Gundy studied the effect of practice on the value of ' $d$ ' for a variety of conditions, including the presentation or withholding of previews of the signal. It appeared that the presentation of previews, which is a common procedure in TSD studies, eliminated the occurrence of practice effects observed under the condition of no previews. The experiments of Swets and Sewall showed practice effects to be dependent upon the level of motivation. They were only detectable with a low level of motivation. Using a forced-choice procedure, Lukaszewski and Elliot and Zwislocki et al. found an increase in the percentage of correct responses in the course of their experiments.

With regard to time on task effects, Deaton, Tobias, and Wilkinson (1971) report a decrease in $\mathrm{d}^{\prime}$ and an increase in $\beta$ over the two halves of a 30-min session. Wilkinson and Seales (1978) recorded an increase in $\beta$ and a nonsignificant decrease in $d^{\prime}$ over the two halves of a session of 240 trials.

Accordingly, there are indications of the existence of practice and time on task effects in detection tasks. The consistency, the extent, and the time course of these effects are more extensively investigated in the present study.

\section{METHOD}

\section{Subjects}

Six students (aged $20-26$ years and including two females), with normal threshold audiograms, were paid for participating in the experiment.

\section{Procedure}

For each subject, the entire experiment consisted of 20 identical sessions. Each session was held on a separate day, with a mean intersession interval of half a week. One session lasted about $1.5 \mathrm{~h}$. A session was composed of four blocks of 200 trials each, short breaks separating the blocks. Before the beginning of each block, five previews were given, that is, signal presentations with a lowered level of noise intensity. Half the trials consisted of a very weak sinusoidal signal $(1,000 \mathrm{~Hz}, 100 \mathrm{msec})$ which was initiated $500 \mathrm{msec}$ after the onset of a 1 -sec burst of wide-band white noise (65 dB SPL). The other half of the trials consisted of a 1-sec noise burst without a signal. The duration of the observation interval was marked by a cue light. The order of presentation of both types of trials was randomized. The intertrial interval was varied randomly between 3 and $5 \mathrm{sec}$. Before the first session, each subject received a training session. Based on the subject's responses in this session, the signal intensity was adjusted to a level that would result in a percentage of correct responses of about $75 \%$. This signal intensity (with a mean of $48 \mathrm{~dB}$ SPL) was maintained throughout the entire experiment. Next, a written instruction was handed to the subject and questions concerning the procedure were briefly answered. The subject responded by pressing one of six buttons, which indicated six categories of confidence, ranging from high confidence that the signal had been presented to high confidence that it had not been presented. Without the subject's knowledge, the latencies of his confidence ratings were recorded from the beginning of the observation interval. No pressure was put on the subject to respond rapidly. During the short pauses between successive blocks of trials, feedback was given by showing the a posteriori function (cf. McNicol, 1972) and discussing any reversal in relation to his rating behavior.

\section{Apparatus and Data Analysis}

The subject was seated in a comfortable chair in a soundattenuated, dimly lit cabin, situated inside a Faraday chamber. The stimuli were generated by a HP 8057 A noise generator and a Krohn-Hite 5200 oscillator and delivered through Sennheiser HMD 110 earphones. The six response buttons were mounted on a panel directly in front of the subject's right hand. A PDP-11/10 computer completely controlled stimulus presentation and response registration, including measurement of response latencies. The analyses of variance (ANOVA) were carried out on the basis of a randomized block factorial design (Kirk, 1968).

\section{RESULTS}

\section{Response Proportions}

Table 1 presents the response proportions averaged over subjects and four successive sessions at a time. In the course of the experiment, a small increase of the hit rate and an almost stable $F A$ rate were observed. ANOVA of the nonparametric sensitivity index $P(A)$ indicated a significant increase of auditory sensitivity $[F(19,95)=2.87, p<.01 ; F$ (linear trend) $(1,95)=34.26, p<.01]$, as shown in Figure 1. This increment corresponds to a rise in $\mathrm{d}^{\prime}$ from .96 to 1.29. 'Given the empirical finding that $\mathrm{d}^{\prime}$ is approximately proportional to signal energy (Green \& Swets, 1966), this improvement is equivalent to a mean increase in signal energy of $1.3 \mathrm{~dB}$ (individual values ranged from .77 to $2.24 \mathrm{~dB}$ ). Because a 6-point rating scale was used, five values of the response bias parameter $\log \beta$ could be calculated. ANOVAs for three of the five measurements showed a significant 
Table 1

Response Proportions for the Six Ratings and the Signal Plus Noise ( $(+n)$ and Noise (n) Presentations

\begin{tabular}{clcccccc}
\hline & & \multicolumn{6}{c}{ Rating } \\
\cline { 3 - 8 } $\begin{array}{c}\text { Ses- } \\
\text { sion }\end{array}$ & & 1 & 2 & 3 & 4 & 5 & 6 \\
\hline \multirow{2}{*}{$1-4$} & $\mathrm{~s}+\mathrm{n}$ & .2248 & .0683 & .0537 & .0269 & .0342 & .0902 \\
& $\mathrm{n}$ & .0536 & .0418 & .0586 & .0559 & .0841 & .2080 \\
$5-8$ & $\mathrm{~s}+\mathrm{n}$ & .2712 & .0564 & .0486 & .0286 & .0323 & .0619 \\
& $\mathrm{n}$ & .0688 & .0477 & .0558 & .0579 & .0860 & .1849 \\
$9-12$ & $\mathrm{~s}+\mathrm{n}$ & .2774 & .0590 & .0363 & .0246 & .0312 & .0700 \\
& $\mathrm{n}$ & .0672 & .0494 & .0434 & .0459 & .0861 & .2095 \\
$13-16$ & $\mathrm{~s}+\mathrm{n}$ & .2758 & .0645 & .0410 & .0209 & .0301 & .0661 \\
& $\mathrm{n}$ & .0582 & .0527 & .0534 & .0474 & .0776 & .2124 \\
$17-20$ & $\mathrm{~s}+\mathrm{n}$ & .2825 & .0701 & .0400 & .0222 & .0303 & .0536 \\
& $\mathrm{n}$ & .0619 & .0542 & .0555 & .0472 & .0811 & .2013 \\
\hline
\end{tabular}

Note-Values are means for all subjects and four successive sessions.

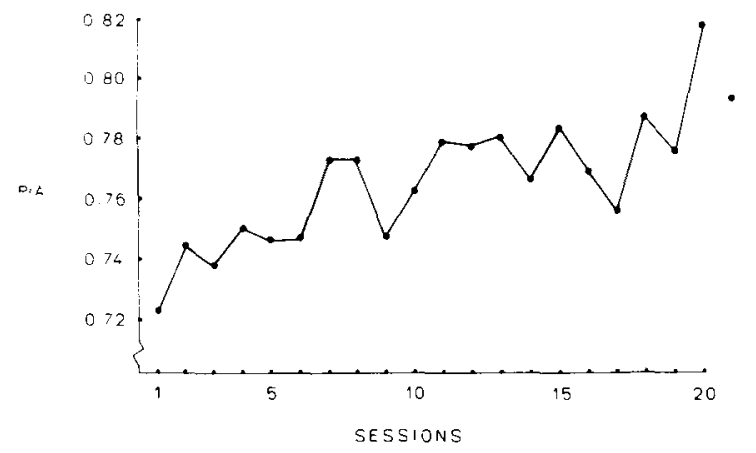

Figure 1. Mean values of $P(A)$ over 20 successive sessions. The 21st value is the mean for five out of the six subjects.

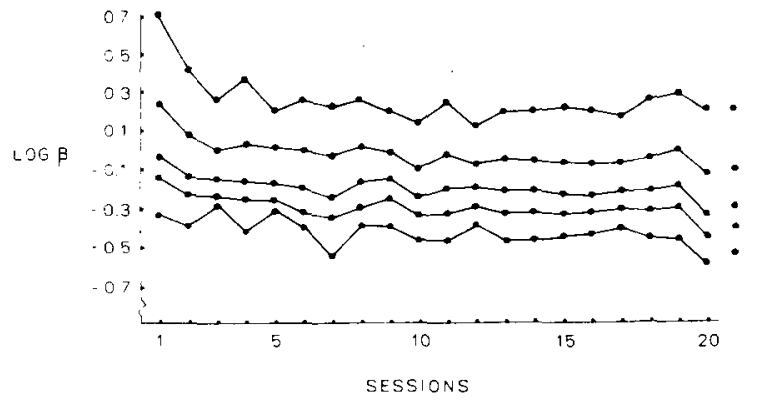

Figure 2. Mean values of $\log \beta$ over 20 successive sessions. The values for the 21st session are based on five out of the six subjects.

decrease over sessions ( $p<.05$ in all cases). Figure 2 shows this decrement to occur during the first half of the experiment.

In Figures 1 and 2, extra points have been added, which were based on data collected from five out of the six subjects 10 days after the end of the experiment. These data clearly are in line with the results reported so far.

Neither the $\mathbf{P}(\mathrm{A})$ nor the $\log \beta$ values showed any interaction between the effects of sessions and blocks. $\mathrm{P}(\mathrm{A})$ showed a significant time on task effect $[F(3,395)=2.92, p<.05]$, which was mainly caused by a decrement over the first two blocks (cf. Figure 3). The values of three out of the five $\log \beta$ measurements increased over blocks; that is, the bias in favor of "yes" responses diminished ( $\mathrm{p}<.05$ in all cases).

\section{Response Latencies}

Table 2 shows response latencies, averaged over subjects and four successive sessions at a time. There appears to be a strong covariation of response latency and confidence rating: for both "yes" and "no" responses, the mean latency decreases with increasing confidence. The table also shows that correct responses are faster than incorrect responses, as are "yes" responses compared with "no" responses (see also Emmerich, Gray, Watson, \& Tanis, 1972).

With regard to the effects of repeated sessions, a significant overall decrease was observed $[\mathrm{F}(18,1135)$ $=5.16, \mathrm{p}<.01 ; \mathrm{F}$ (linear trend $)(1,1135)=23.29$, $\mathrm{p}<.01]$. Although all ratings showed a similar

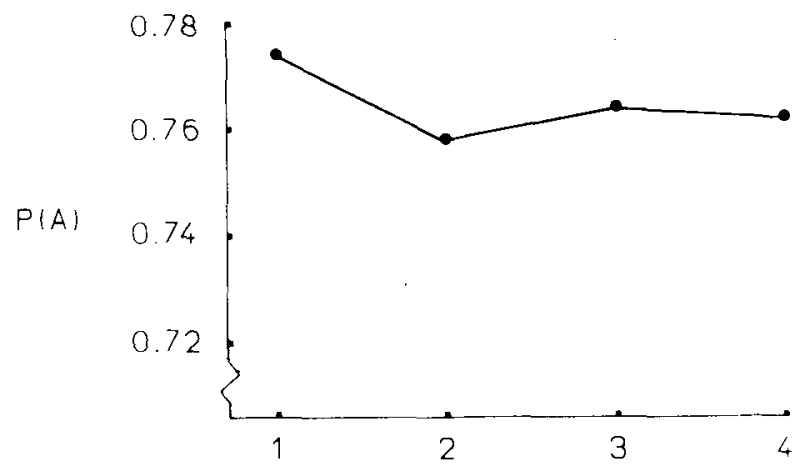

BLOCKS

Figure 3. Mean values of $P(A)$ over the four successive blocks per session.

Table 2

Response Latencies (in Milliseconds) for the Six Ratings and the Signal Plus Noise (s+n) and Noise (n) Presentations

\begin{tabular}{crrrrrrr}
\hline \multirow{2}{*}{$\begin{array}{l}\text { Ses- } \\
\text { sion }\end{array}$} & & \multicolumn{6}{c}{ Rating } \\
\cline { 3 - 8 } $2-4$ & 1 & 2 & 3 & 4 & 5 & 6 \\
\hline \multirow{2}{*}{$5-8$} & n & 516 & 717 & 830 & 1005 & 914 & 1288 \\
& stn & 526 & 738 & 799 & 932 & 799 & 806 \\
& n & 637 & 705 & 795 & 869 & 801 & 652 \\
$9-12$ & stn & 523 & 727 & 733 & 850 & 754 & 609 \\
& n & 588 & 754 & 748 & 781 & 751 & 581 \\
$13-16$ & stn & 486 & 646 & 691 & 796 & 661 & 575 \\
& n & 589 & 628 & 697 & 707 & 648 & 538 \\
$17-20$ & stn & 490 & 609 & 707 & 832 & 702 & 567 \\
& n & 561 & 634 & 644 & 670 & 645 & 533 \\
\hline
\end{tabular}

Note-Values are means for all subjects and four successive sessions. Due to apparatus failure, the data for the first session are lacking. 
trend, the latencies of the medium ratings decreased most. Thus, in the course of the experiment, there was a substantial decrease of the absolute latency differences between the ratings.

\section{DISCUSSION}

The results of this study show that detection performance as measured by the sensitivity index $P(A)$ in a simple detection task improves gradually over more than 16,000 trials. During the first half of the experiment, an increasing bias towards "yes" responses was also observed. The substantial decrement of the response latencies indicated that these effects were not mediated by changes in the tradeoff between accuracy and speed.

Within the framework of TSD, only short-term practice effects have been reported, with the exception of the study of Whitmore, Ermey, and Williams (1968), who recorded for one subject an improvement of $3 \mathrm{~dB}$ over 10,000 trials. Short-term practice effects were also found in a previous experiment of ours (Kerkhof, Schaaf, \& Korving, Note 1) which employed a 1,900-msec foreperiod, no cue light, and an 8-point rating scale. Over some 1,500 trials, the mean improvement amounted to $2.3 \mathrm{~dB}$. The comparison of these results with those of others (see introduction) suggests that these short-term practice effects are largely task-specific and only indirectly related to the detection process. In the study just cited, these task-specific effects most probably consisted of a gradually improving time estimation of the moment of signal occurrence. Time uncertainty has been shown to exert a deteriorating effect upon detection performance (cf. Egan, Greenberg, \& Schulman, 1961). Similar task variables probably can also account for a substantial part of long-term practice effects as observed in complex tasks, such as fine temporal judgments (Tanner \& Rivette, 1963) and the detection of interaural signal differences (Hafter $\&$ Carrier, 1970). However, the role of these effects in the simple TSD task of this study is questionable.

The second major finding of this study concerns the effects of time on task, that is, a decrease in $\mathrm{P}(\mathrm{A})$ and an increase in $\log \beta$ over blocks. These trends are similar to those found in the TSD studies of Deaton et al. (1971) and Wilkinson and Seales (1978). The validity of these results-particularly with regard to the values of $\log \beta$-rests upon the assumption of equal and stable variances of the noise and signal-plus-noise response distributions. In the event of unequal variances, $\beta$ is known not to be monotonous with the evidence value of the observation (Green \& Swets, 1966). For the present data, the ratio of Ssn/Sn proved invariant over sessions, but not so over blocks $[F(3,395)=3.25, p<.05]$. The mean values decreased, particularly from the first to the second block (mean values: $1.48,1.37,1.34,1.32$ ).
The conditions of a decreasing sensitivity and a changing $\mathrm{Ssn} / \mathrm{Sn}$ ratio might have compelled the observer to adjust his response bias in order to maintain stable cutoff points on the evidence scale. This was verified by analyzing the values of $z_{n}$, a measure of the criterion cutoff, as a function of blocks. As a result, no significant effects were found $(p>.10$ in all cases). It therefore appears that the block effects upon the values of $\log \beta$ are secondary to variations of the $\mathrm{Ssn} / \mathrm{Sn}$ ratio.

\section{REFERENCE NOTE}

1. Kerkhof, G. A., Schaaf, T. W, van der, \& Korving, J. Practice effects in an auditory signal detection task (Internal Report E044-78). Leiden, The Netherlands: Department of Experimental Psychology, University of Leiden, 1978.

\section{REFERENCES}

Deaton, M., Tobias, J. S., \& Wilkinson, R. T. The effect of sleep deprivation on signal detection parameters. Quarterly Journal of Experimental Psychology, 1971, 23, 449-452.

Egan, J. P., Greenberg, G. Z., \& Schulman, A. I. Interval of time uncertainty in auditory detection. Journal of the Acoustical Society of America, 1961, 33, 771-778.

Emmerich, D. S., Gray, J. L., Watson, C. S., \& Tanis, D. C. Response latency, confidence, and ROCs in auditory signal detection. Perception \& Psychophysics, 1972, 11, 65-72.

Green, D. M., \& SwETS, J. A. Signal detection theory and psychophysics. New York: Wiley, 1966.

Gundy, R. F. Auditory detection of an unspecified signal. Journal of the Acoustical Society of America, 1961, 33, 1008-1012.

Hafter, E. R., \& Carrier, S. C. Masking-level differences obtained with a pulsed tonal masker. Journal of the Acoustical Society of America, 1970, 47, 1041-1047.

Kıнк, R. E. Experimental design: Procedures for the behavioral sciences. Belmont, Calif: Brooks/Cole, 1968.

Lukaszewski, J. S., \& Elliott, D. N. Auditory threshold as a function of forced-choice techniques, feedback and motivation. Journal of the Acoustical Society of America, 1962, 34, 223-228.

McNicol, D. A primer of signal detection theory. London: Allen \& Unwin, 1972.

Swets, J. A., \& Sewall, S. T. Invariance of signal detectability over stages of practice and levels of motivation. Journal of Experimental Psychology, 1963, 66, 120-126.

Tanner, W. P., Jr., \& Rivette, C. L. Learning in psychophysical experiments. Journal of the Acoustical Society of America, 1963, 35, 1896 (N4).

Whitmore, J. K., Ermey, H. L., \& Williams, P. Some results bearing on the stability of psychometric data. Journal of the Acoustical Society of America, 1968, 44, 370.

Wilkinson, R. T., \& Seales, D. M. EEG event-related potentials and signal detection. Biological Psychology, 1978, 7, 13-28.

Zwislocki, J., Maire, F., Feldoman, A. S., \& Rubin, H. On the effect of practice and motivation on the threshold of audibility. Journal of the Acoustical Society of America, 1958, 30, 254-262.

\section{NOTE}

1. The $\mathrm{d}^{\prime}$ value of 1.29 for the 21 st session is more in line with the trend of the data than is the extreme value of the 20th session, which was probably influenced by motivational factors (payday). accepted March 6, 1980.) 